\title{
DEEP MULTI-VIEW FEATURES FROM RAW AUDIO FOR ACOUSTIC SCENE CLASSIFICATION
}

\author{
Arshdeep Singh, Padmanabhan Rajan, Arnav Bhavsar \\ MANAS LAB, School of Computing and Electrical Engineering, \\ Indian Institute of Technology, (IIT), Mandi, India \\ d16006@students.iitmandi.ac.in, [padman,arnav]@iitmandi.ac.in
}

\begin{abstract}
In this paper, we propose a feature representation framework which captures features constituting different levels of abstraction for audio scene classification. A pre-trained deep convolution neural network, SoundNet, is used to extract the features from various intermediate layers corresponding to an audio file. We consider that the features obtained from various intermediate layers provide the different types of abstraction and exhibits complementary information. Thus, combining the intermediate features of various layers can improve the classification performance to discriminate audio scenes. To obtain the representations, we ignore redundant filters in the intermediate layers using analysis of variance based redundancy removal framework. This reduces dimensionality and computational complexity. Next, shift-invariant fixed-length compressed representations across layers are obtained by aggregating the responses of the important filters only. The obtained compressed representations are stacked altogether to obtain a supervector. Finally, we employ the classification using multi-layer perceptron and support vector machine models. We comprehensively perform the validation of the above assumption on two public datasets; Making Sense of Sounds and open set acoustic scene classification DCASE 2019.
\end{abstract}

Index Terms- Acoustic scene classification, Deep neural network, SoundNet.

\section{INTRODUCTION}

Acoustic scene classification (ASC) aims to utilize the audio information in everyday soundscapes to recognise the underlying physical environment (commonly referred to as scene). Traditionally, most of the work in audio scene classification, inspired from the closely related fields such as speech recognition and music analysis, employed hand-crafted time-frequency based representations such as spectrogram, log-mel energy, mel-frequency cepstral coefficients, constant-Q-transform etc. However, the hand-crafted features are often not able to adapt to acoustic scenes data owing to the complexity which arises mostly from many independent unknown sources which produces unstructured sounds. Moreover, the audio information in the scene spans whole audio spectrum. To circumvent this, feature learning based approaches are being applied to learn relevant information directly from time-frequency representations. For examples, the work in [1] applied matrix factorization based representations. [2] used i-vector and deep convolution neural network $(\mathrm{CNN})$ based features. The study [3] used a dictionary learning framework which captures the rare and most frequently occurring sound events. Apart from this, ensemble based methods which combines multiple channels and models are also being reported [4].

A few studies explored the feature representations from raw audio directly. For example, [5] demonstrated that deep CNNs trained directly on very long raw acoustic sound waveforms can outperform than CNNs with similar architecture on handcrafted features. The study [6] proposed a pre-trained deep convolutional neural network, SoundNet, that accepts raw audio as input. The work [7] performed a layer-wise analysis on SoundNet layers and proposed an ensemble framework in decision space.

In this paper, we propose a representations framework for ASC by utilizing the intermediate layer representations obtained using SoundNet, from raw audio. Our underlying assumption is that the intermediate representations of different layers in SoundNet, correspond to different details of an audio. To illustrate this, we show the frequency response for some of the learned filters in the first and second convolution layers of SoundNet in Figure 1. It can be observed that the filters (a) and (b) in the first convolution layer have different bandpass frequency characteristics and hence, produce different details of an audio. In the subsequent layers, the output of the filters from the previous layer is being operated with a different set of filters, which also posses different bandpass information. As shown in the Figure 1, the learned filters (c)-(e) in the second convolution layer have different bandpass characteristic and operate on the output of the filter (a) (learned in the first convolution layer). Similarly, the filters (f)-(h) in the second convolution layer operate on the output of the filter (b) which is being learned in the first convolution layer. Therefore, an audio signal is operated upon by filters having different frequency responses along the layers. Moreover, the non-linear operations such as batch normalization and ReLU transformation, project the data into different subspaces [8]. Therefore, the representations obtained from different layers can be considered as exhibiting different characteristics of an audio.

Henceforth, such intermediate representations are further transformed into compressed features as explained in subsection 2.2 and concatenated altogether to build a supervector, which captures the multiple details of an audio. Empirically, we analyse the validity of the proposed feature representation approach for two publicly available datasets.

The rest of the paper is organized as follows. Section 2 gives the main idea of our proposed method in which we describe the feature extraction, the compressed feature representation and the classification methods. Section 3 shows the experimental setup and findings. In Section 4, we conclude this paper. 


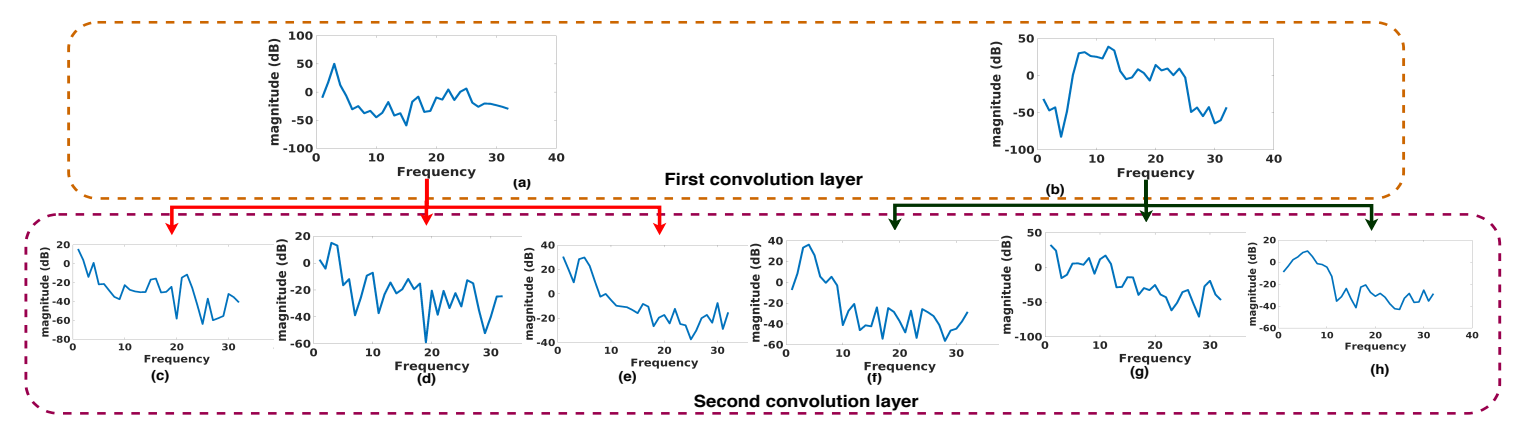

Figure 1: Single-sided magnitude of frequency spectrum for some of the learned filters in SoundNet. (a) and (b) shows the frequency response for fifth and sixteenth filters respectively in the first convolution layer. (c)-(h) shows frequency response of filters in second convolution layer. The filters (c)-(e) (here, only three are shown) operate on the output produced by filter (a). Similarly, (f)-(h) shows frequency response for filters which operate on response of (b) filter. Here, the frequency spectrum is computed using 64-point DFT and the magnitude (dB) is $20 \log |X(f)| .|X(f)|$ is the magnitude of frequency spectrum.

\section{PROPOSED METHODOLOGY}

\subsection{A brief on pre-trained 1-D CNN}

SoundNet is a 1-D CNN, trained on large-scale weakly labeled video datasets, ultimately performing transfer learning from video to audio. The architecture has 8-layers namely convolution, pooling layers and operates on the raw audio directly. Each convolution layer (denoted as C) output is computed by convolution operation followed by batch normalization (denoted as $\mathrm{p}-\mathrm{C}$ ) and non-linear activation operations (ReLU).

An audio signal $x$, of duration $t$ seconds, and sampled at $f_{s}$ frequency, can be represented into a 2-D representations $\in \mathbb{R}^{N \times s}$ using any intermediate layer of SoundNet. Here, $N$ and $s$ represent the number of feature maps and their size respectively in a given layer.

\subsection{Compressed feature representation and classification}

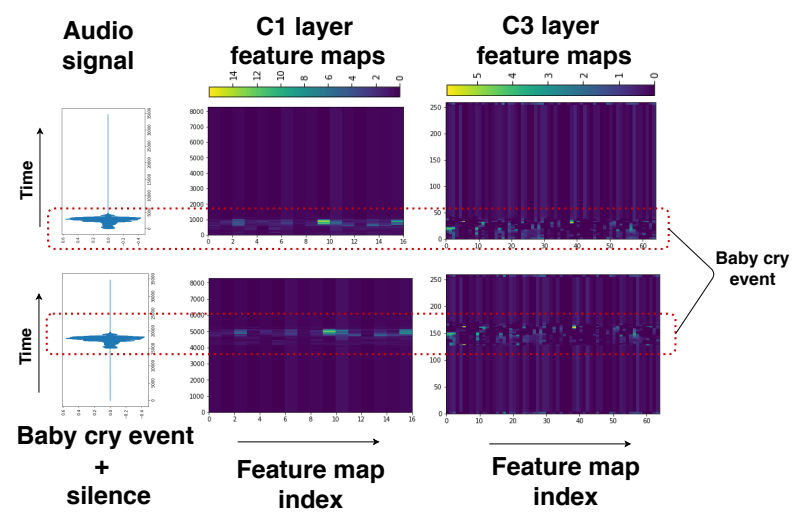

Figure 2: 2-D intermediate layer representations for first (C1) and third (C3) convolutional layer in SoundNet corresponding to $5 \mathrm{sec}-$ onds audio. Here, the audio is a baby cry event and a silence.

The intermediate 2-D feature representations obtained from SoundNet have very high dimensionality of the order of approx. $16 \mathrm{M}$ and $27 \mathrm{k}$ for an audio of length 5 seconds sampled at $44.1 \mathrm{kHz}$ for first (C1) and third (C3) convolution layer respectively. In addition, the size of representations depend on the input audio length and the representations are not time-invariant. Figure 2 demonstrates the time-variance of the intermediate layers representations as the input shifts in time.

We reduce the dimensionality in two ways: first, since all the learned filters in SoundNet do not provide discriminatory response [9], some of the filter responses can be ignored. We employ analysis of variance method based pruning procedure as proposed in [10] to identify the filters, which generates discriminating response across scene classes. This is done for each of layers independently. Ignoring the non-discriminating responses result into a reduced dimension 2-D representations $\in \mathbb{R}^{N^{\prime} \times s}$, where $N^{\prime} \leq N$. Second, to compute the time-invariant representations and compress the intermediate representation further, global sum pooling is applied across the response of filters. This results into a fixed-length representations $\in \mathbb{R}^{N^{\prime}}$ of an audio for a particular intermediate layer. Henceforth, we call these fixed-length representations as compressed features.

We utilize the compressed features from various layers to build a global super-vector $\xi$, representing different details of an audio by concatenating the compressed features from various layers. A variable length audio of very high dimensionality can now be represented using $\xi$-features. Since these features represent different characteristics of an audio, therefore we call them "multi-view features". Finally, we employ multilayer perceptron (MLP) model and support vector machine (SVM) as a classifier. The flow diagram of the overall proposed framework is shown in Figure 3.

\section{PERFORMANCE EVALUATION}

\subsection{Dataset and Experimental setup}

We use the following audio scene classification (ASC) datasets for evaluation: first, the Making Sense of Sounds (MSOS) challenge dataset [11], comprising of a development dataset consists of 1500 audio files divided into the five categories, each containing 300 files. The number of different sound types within each category is not balanced. The evaluation dataset consists of 500 audio files, 100 files per category. F-measure and accuracy metrics are used to measure the performance.

Second, the TAU Urban Scenes openset development and 


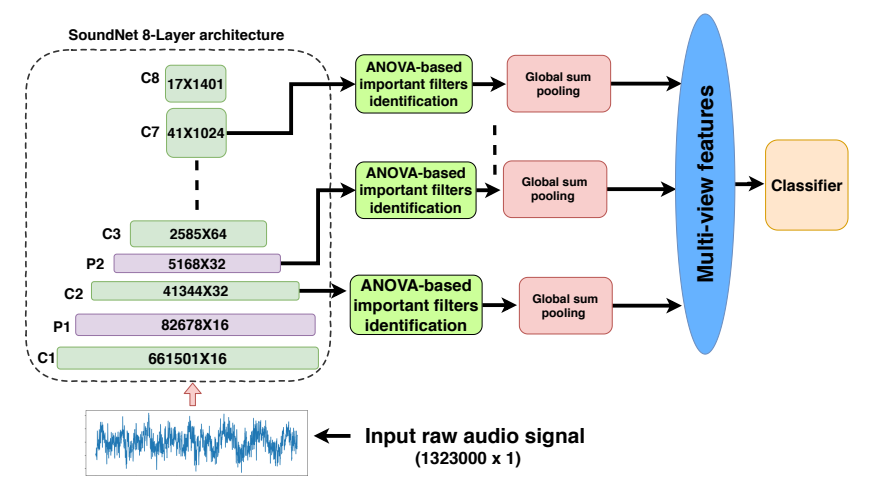

Figure 3: Overall proposed framework. The size and number of feature maps for each layer are shown corresponding to input of 30 seconds length sampled at $44.1 \mathrm{kHz}$. C1, P1, C2 etc. represents first convolution, first pooling, second convolution and so on layers.

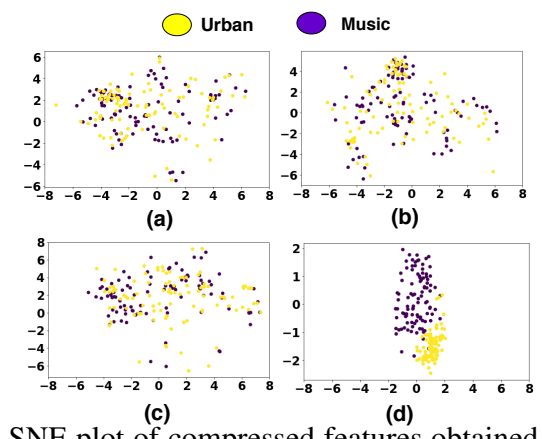

Figure 4: t-SNE plot of compressed features obtained from (a) P2, (b) C3 (c) C7 and (d) the proposed multi-view features from various layers of two classes namely, urban and music from MSOS Evaluation dataset.

leaderboard dataset [12] (DCASE), comprising of ten known target classes and one unknown class is used. The "unknown" class has additional data of several unknown acoustic scenes. The training and testing development data is divided as described in the task protocol for all 11 classes. We handle the out-of-set classification as follows. A given test recording is being classified as a particular scene class if the class-specific probability is greater than threshold $(\tau), \tau \in[0,1)$. Otherwise, if all classes have lesser than $\tau$, the sample is assigned as an unknown class label. For DCASE dataset, the weighted average accuracy $\left(\alpha_{w}\right)$, the accuracy of known classes $\left(\alpha_{k}\right)$ and unknown class $\left(\alpha_{u}\right)$ is used as metric as given in the Equation 1 . We report the performance for leaderboard dataset using the online portal.

$$
\alpha_{w}=0.5 * \alpha_{k}+0.5 * \alpha_{u}
$$

The compressed features are computed from C2 to C7 layer (a total of 4256 filter responses) including the $\mathrm{p}-\mathrm{C}$ layers. We obtain a total of 1307 non-redundant filter responses, as explained in section 2.2. This leads to give multi-view feature $\xi \in \mathbb{R}^{1307}$ obtained from 12 intermediate layers after performing global sum pooling on each filter response.

The classification model parameters such as number of hidden layers, neurons in MLP and hyper-parameters of non-linear SVM are selected empirically. The MLP is trained with Adam optimizer

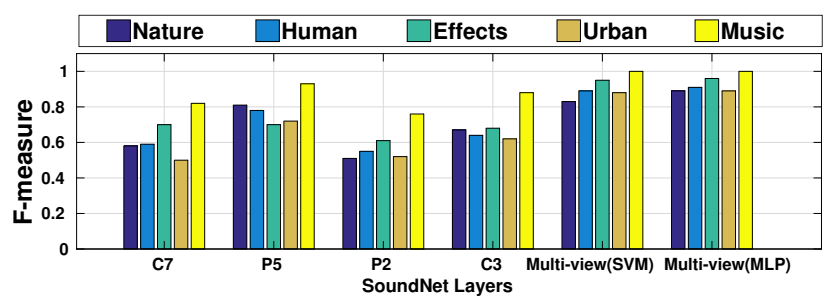

Figure 5: F-measure obtained with compressed features from C7, P5, P2, C3 layers using SVM and multi-view features using SVM and MLP for MSOS dataset.

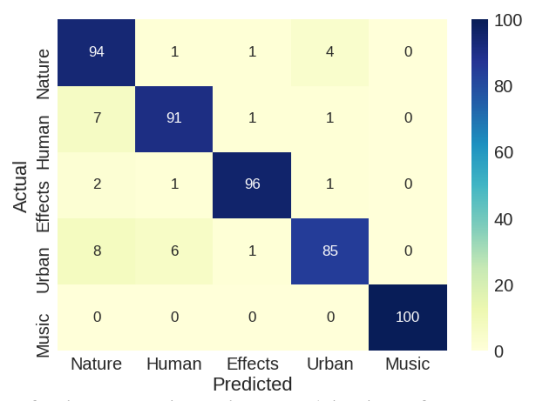

Figure 6: Confusion matrix using multi-view features with MLP for MSOS dataset.

and cross-entropy categorical loss for 100 epochs. Empirically, we find that single hidden layer having 30 neurons with hyperbolic tangent activation function suits well for our classification task.

\subsection{Results and Analysis}

\subsubsection{Dataset (A): Making sense of Sounds}

Figure 4 shows the t-SNE plot for compressed features obtained from various intermediate layers and $\xi$-features for two sound classes. It can be observed that the $\xi$-feature space shows lesser inter-class overlap as compared to the feature space generated from the individual intermediate layers.

In addition to give more separability, the $\xi$-feature space also utilizes complementary information given by various layers. This can be observed from Figure 5, which shows the F-measure, computed using the compressed features obtained from various intermediate layers and $\xi$-features. The F-measure for a given class varies across layers, for example, "Nature" has larger F-measure for C3 layer as compared to C7 and P2 layers. This is valid for "Human" as well. However, C7 layer has larger F-measure for "Effects" as compared to P2 and C3 layers. This empirically shows that the feature space generated from various layers constitute complementary information. Utilizing the compressed features from various layers to build multi-view features improve the F-measure of all scene class significantly. The accuracy for compressed features from $\mathrm{C} 7$, $\mathrm{P} 5, \mathrm{P} 2, \mathrm{C} 3$ and $\xi$-features using SVM is $64.2 \%, 79 \%, 59.6 \%, 70 \%$ and $91 \%$ respectively. Using MLP, the accuracy obtained with $\xi$ features is $93.2 \%$. Figure 6 gives the confusion matrix obtained using $\xi$-features with MLP as a classifier. It can be observed that "Urban" is most frequently confused as "Nature" and "Human".

Comparison with existing methods: Figure 7 shows the comparison of class-wise accuracy for baseline, state-of-the-art [11] and 


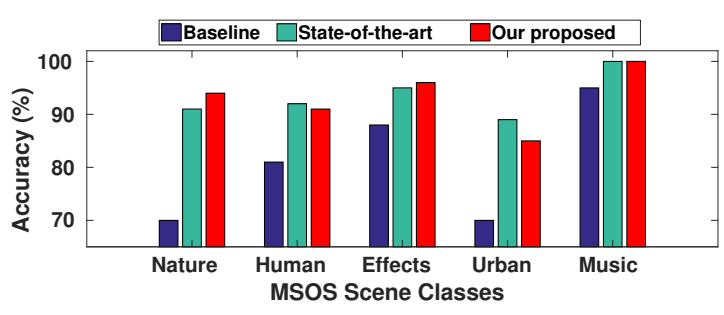

Figure 7: Comparison of class-wise accuracy with the existing methods for MSOS dataset.
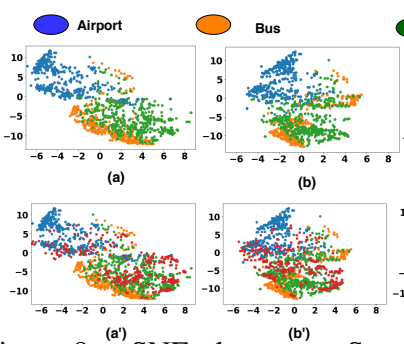

(b)
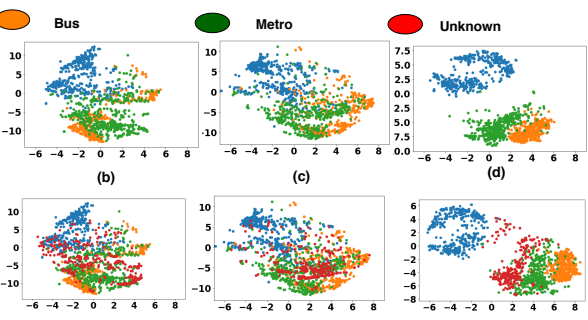

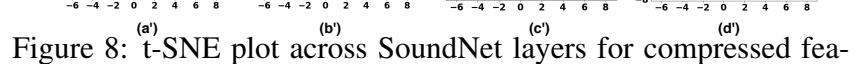

Figure 8: $\begin{gathered}\left(\mathrm{a}^{\prime}\right) \\ \mathrm{t}-\mathrm{SNE} \text { plot across SoundNet layers for compressed fea- }\end{gathered}$ tures from (a) P2 (b) C3 (c) C7 and (d) the proposed multi-view features for three scene class, namely airport, bus and metro. Here, (a'), (b'), (c') and (d') shows the same when unknown class is also being considered.

our proposed approach with MLP. For all classes, our proposed approach provides significant improvement as compared to the baseline. On the other hand, the performance is equivalent to state-ofthe-art with an improvement for "Nature" and Effects by $3 \%$ and 1\% respectively, however, the performance degrades for "Human" and "Urban" by $1 \%$ and $4 \%$ respectively. The accuracy across classes for baseline, the state-of-the-art and our proposed approach is $81 \%$, $93.4 \%$ and $93.2 \%$ respectively.

\subsubsection{Dataset (B): TAU Urban Acoustic Scenes 2019 Openset}

Figure 8 shows the t-SNE plot obtained using compressed features from various layers and $\xi$-features for three known classes, airport, bus, metro and one unknown class. It is notable that the proposed multi-view features are able to provide better separation among known classes as compared to the classes considering both known and unknown.

Figure 9 gives the performance obtained using MLP and SVM classifier as $\tau$ varies from 0 to 1 . For $\tau$ close to 0 , the classifiers are able to classify the known classes significantly well. However, the unknown class has poor accuracy. As $\tau$ increases, the unknown class accuracy increases, however, when $\tau$ is very close to 1 , the known class accuracy is poor. It can be observed that there is a trade-off in accuracy of the known and unknown class with threshold. Our proposed framework improves $\alpha_{w}$ significantly by $12 \%$ to $31 \%$ (choosing $0.5<\tau<1$ ) as compared to that of baseline [12] which has $\alpha_{w}$ equals to $48.7 \%$.

For leaderboard dataset, $\alpha_{w}$ is computed through the public leaderboard online portal. Figure 10 shows $\alpha_{w}$ as a function of $\tau$ with MLP and SVM classifiers. In case of MLP, as the $\tau$ increases towards 1 , the $\alpha_{w}$ also increases and approaches to the baseline performance which is around $44 \%$ (private leaderboard). Utilizing SVM, the $\alpha_{w}$ remains constant at $17.5 \%$ beyond 0.5 threshold. This may be due to the over-fitting of the SVM classifier towards the
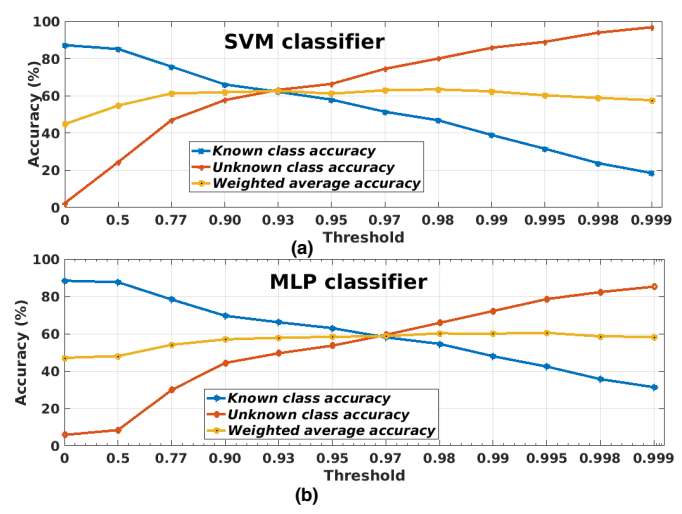

Figure 9: Known, unknown and weighted average accuracy as a function of threshold ( $\tau$ ) using (a) SVM and (b) MLP as a classifier for DCASE development dataset.

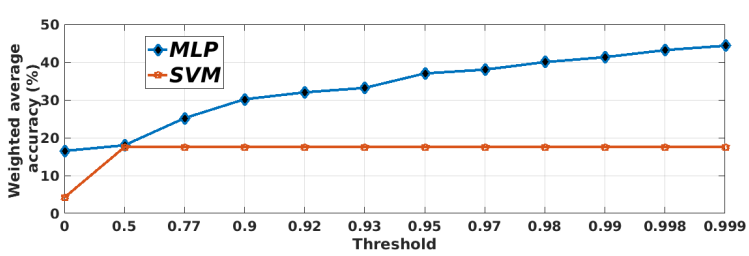

Figure 10: Weighted average accuracy $\left(\alpha_{w}\right)$ as a function of threshold using MLP and SVM for DCASE leaderboard dataset.

training dataset. The predicted scene labels obtained using different threshold for leaderboard dataset can be found on this link.

\subsubsection{Discussion}

The proposed approach is performing well for MSOS dataset. However, the overall performance for DCASE dataset especially the leaderboard dataset (recorded at different locations and time instants), is not that overwhelming. We speculate that this might be caused because the resulting latent space obtained from a pretrained model is not able to discriminate each of the classes, especially the "unknown" class. SoundNet is trained using transfer learning from 2 million Flicker videos [6]. The MSOS dataset contains the audio files collected from Freesound and the other online sources [11]. This may lead to give similar distributions between the learned parameters of SoundNet and the MSOS dataset, hence, the model shows good representation strength. However, the DCASE dataset is recorded at various locations in an uncontrolled environment and with more confusing classes. Hence, DCASE dataset shows more domain mismatch to the pre-trained SoundNet. In addition, the complexity of DCASE dataset can not be ignored. Therefore, we experiment to adapt the existing model with new datasets such as DCASE and expecting to perform better than the approach proposed in this paper in future.

\section{CONCLUSION}

We propose a feature representation framework using various intermediate levels of the pre-trained deep CNN SoundNet, for acoustic scene classification. The combined features from the intermediate layers are able to provide better discrimination as compared to the features from each of the individual layers. 


\section{REFERENCES}

[1] V. Bisot, R. Serizel, S. Essid, and G. Richard, "Supervised nonnegative matrix factorization for acoustic scene classification,” DCASE2016 Challenge, Tech. Rep., September 2016.

[2] H. Eghbal-Zadeh, B. Lehner, M. Dorfer, and G. Widmer, "CPJKU submissions for dcase-2016: a hybrid approach using binaural i-vectors and deep convolutional neural networks," IEEE AASP Challenge on Detection and Classification of Acoustic Scenes and Events (DCASE), 2016.

[3] A. Singh, A. Thakur, and P. Rajan, "APE: Archetypalprototypal embeddings for audio classification," in 2018 IEEE 28th International Workshop on Machine Learning for Signal Processing (MLSP). IEEE, 2018, pp. 1-6.

[4] S. Mun, S. Park, D. Han, and H. Ko, "Generative adversarial network based acoustic scene training set augmentation and selection using SVM hyper-plane,' DCASE2017 Challenge, Tech. Rep., September 2017.

[5] T. Purohit and A. Agarwal, "Acoustic scene classification using deep CNN on raw-waveform," DCASE2018 Challenge, Tech. Rep., September 2018.

[6] Y. Aytar, C. Vondrick, and A. Torralba, "Soundnet: Learning sound representations from unlabeled video," in Advances in neural information processing systems, 2016, pp. 892-900.

[7] A. Singh, A. Thakur, P. Rajan, and A. Bhavsar, "A layer-wise score level ensemble framework for acoustic scene classification," in 2018 26th European Signal Processing Conference (EUSIPCO). IEEE, 2018, pp. 837-841.

[8] S. Yu and J. C. Principe, "Understanding autoencoders with information theoretic concepts," Neural Networks, vol. 117, pp. 104-123, 2019.

[9] A. RoyChowdhury, P. Sharma, E. Learned-Miller, and A. Roy, "Reducing duplicate filters in deep neural networks," in NIPS workshop on Deep Learning: Bridging Theory and Practice, vol. 1, 2017.

[10] A. Singh, P. Rajan, and A. Bhavsar, "Deep hidden analysis: A statistical framework to prune feature maps," in ICASSP 20192019 IEEE International Conference on Acoustics, Speech and Signal Processing (ICASSP). IEEE, 2019, pp. 820-824.

[11] C. Kroos, O. Bones, Y. Cao, L. Harris, P. J. Jackson, W. J. Davies, W. Wang, T. J. Cox, and M. D. Plumbley, "Generalisation in environmental sound classification: the making sense of sounds data set and challenge," in ICASSP 2019-2019 IEEE International Conference on Acoustics, Speech and Signal Processing (ICASSP). IEEE, 2019, pp. 8082-8086.

[12] A. Mesaros, T. Heittola, and T. Virtanen, "A multi-device dataset for urban acoustic scene classification," in Proceedings of the Detection and Classification of Acoustic Scenes and Events 2018 Workshop (DCASE2018), November 2018, pp. 9-13. [Online]. Available: https://arxiv.org/abs/1807.09840 